\title{
Chromatin forms nanoscale three-dimensional packing domains with fractal-like scaling behavior in vitro
}

Yue Li ${ }^{1,+}$, Vasundhara Agrawal ${ }^{2,+}$, Ranya K. A. Virk ${ }^{2,+}$, Eric Roth ${ }^{3}$, Adam Eshein ${ }^{2}$, Jane Frederick$^{2}$, Kai Huang ${ }^{4}$, Luay Almassalha ${ }^{5}$, Reiner Bleher ${ }^{3}$, Marcelo A. Carignano ${ }^{2}$, Igal Szleifer ${ }^{2,6}$, Vinayak P. Dravid ${ }^{3, *}$, Vadim Backman ${ }^{2, *}$

${ }^{1}$ Applied Physics Program, Northwestern University, Evanston, Illinois 60208, USA.

${ }^{2}$ Department of Biomedical Engineering, Northwestern University, Evanston, Illinois 60208, USA.

${ }^{3}$ Department of Materials Sciences and Engineering, Northwestern University, Evanston, Illinois 60208, USA.

${ }^{4}$ Institute of Systems and Physical Biology, Shenzhen Bay Laboratory, Shenzhen 518132, China

${ }^{5}$ Medical Scientist Training Program, Feinberg School of Medicine, Northwestern University, Evanston, Illinois 60611, USA.

${ }^{6}$ Department of Chemistry, Northwestern University, Evanston, Illinois 60208, USA.

${ }^{+}$These authors contributed equally to the manuscript.

*Correspondence and requests for materials should be addressed to V.B. (email: $\underline{\mathrm{v}}-$ backman@northwestern.edu) or V.P.D. (email: v-dravid@ northwestern.edu)

\begin{abstract}
Chromatin organization over a wide range of length scales plays a critical role in the regulation of gene expression and deciphering these processes requires high-resolution, three-dimensional, quantitative imaging of chromatin structure in vitro. Herein we introduce ChromSTEM, a method that utilizes high angle annular dark-field imaging and tomography in scanning transmission electron microscopy combined with DNA-specific staining for electron microscopy. We utilized ChromSTEM to quantify chromatin structure in cultured cells and the scaling behavior of the chromatin polymer. We observed that chromatin forms spatially well-defined nanoscale domains which adopt a mass fractal internal structure up to around $100 \mathrm{~nm}$ in radius, with a radially decreasing mass-density from the center to the periphery. The morphological properties of the domains vary within the same cell line and seem to exhibit greater heterogeneity across cell lines, which might indicate how chromatin organization regulates gene expression.
\end{abstract}

\section{Introduction}

Three-dimensional chromatin packing in the cell nucleus plays an important role in regulating numerous cellular processes and large-scale alterations in chromatin structure are associated with cancer, neurological and autoimmune disorders, and other complex diseases (1-3). The fundamental repeating unit of chromatin is the nucleosome, in which $147 \mathrm{bp}$ of the DNA is wrapped around a core histone octamer (4). The core particle adopts a squat cylindrical shape, with a diameter and height of approximately $11 \mathrm{~nm}$ and $5.5 \mathrm{~nm}$, respectively (5). The nucleosome is the first level of higher-order packing of the chromosomal DNA. Nucleosomes are connected by linker DNA, which altogether form what is referred to as the 10 -nm chromatin fiber (6). A long-standing paradigm holds that the $10-\mathrm{nm}$ chromatin fiber continuously folds into a more condensed structure to varying degrees until the chromosomal level of compaction is achieved $(7,8)$. Central to the textbook view of chromatin packing is that 10 -nm chromatin fibers assemble into 30 -nm fibers, 
that further fold into 120 -nm chromonema, to 300 - to $700-\mathrm{nm}$ chromatids, and ultimately, mitotic chromosomes (9-12).

However, the key tenant of this view, the 30-nm fiber, has been challenged by an abundance of recent evidence. Various studies using cryo-electron microscopy, small-angle X-ray scattering, electron spectroscopy imaging, and super-resolution microscopy failed to observe 30-nm fibers in interphase chromatin or mitotic chromosomes in numerous cell lines (13-16). Recently, a combination of DNA-specific staining (ChromEM) and multi-tilt electron tomography (ChromEMT) observed in situ that the chromatin fiber consists of disordered chains that have diameters between 5 to $24 \mathrm{~nm}$ during both interphase and mitosis, with a higher packing concentration in mitotic chromosomes (17). Together, these studies suggest that the chromosome organization is constructed by 10 -nm fibers without folding into $30-\mathrm{nm}$ fibers $(14,18,19)$. In this new paradigm, the 10-nm fibers condensate into highly disordered and interdigitated states, which may be constantly moving and rearranging at the local level (20-22).

Despite their dynamic and fluid-like nature, several complementary studies have revealed distinct, large-scale, domain-like chromatin structures. Recently, photoactivated localization microscopy (PALM) live-cell imaging observed nucleosomes are arranged in physically compact chromatin domains with a diameter of around $160 \mathrm{~nm}$ in mammalian cells (23). 3D-structured illumination microscopy (SIM) imaging showed that DNA labeled with fluorescent in situ hybridization (FISH) forms chromatin domain clusters (CDCs) of around 120 to $150 \mathrm{~nm}$ in diameter with radially arranged layers of increasing chromatin compaction from the periphery towards the CDC core for mammalian cells (24). Meanwhile, chromatin conformation capture (3C) and related methods (4C, $5 \mathrm{C}$, Hi-C, Dip-C) have revealed that the eukaryotic genome is partitioned into topologically associating domains (TADs) at the scale of several hundreds of kilobases (kbs) and smaller loop domains (25-28). Recently, high-resolution imaging combined with Oligopaint staining traced the TADs identified by $\mathrm{Hi}-\mathrm{C}$ in single cells, providing a link between the nanoscopic spatial structures and genomic domains $(29,30)$. Combining both in vivo and in vitro data, such domain structure may represent fundamental building blocks used to assemble higher-order compartments, and ultimately an interphase chromosome partitioned into euchromatin and heterochromatin. However, the understanding of the internal structure of the chromatin domains is currently lacking, and new experiments with higher resolution such as ChromEMT are needed.

In parallel with these experimental findings, many models have been proposed to understand the folding of the domain-like chromatin structure. A fractal globule model that describes chromatin as a collapsed polymer where topological constraints result in a hierarchy of non-entangled structures, explains earlier Hi-C results but was later challenged by data with higher resolution $(31,32)$. Additionally, chromatin domains observed by recent PALM imaging adopt a folding deviation from the fractal globule model at large length scales (33). More recently, a logarithmic fractal model was proposed to describe the large-scale organization of chromatin based on smallangle-neutron-scattering (SANS) experiments (34). Additional statistical models of chromatin have also been proposed, including the novel self-returning random walk (SRRW) model depicts chromatin as non-globular, porous, and irregular "tree" domains and is able to reproduce key experimental observations including TAD-like features observed in Hi-C contact maps (35). 
However, the exact model to understand the real chromatin organization is still largely unknown because structural analysis of individual chromatin domains at single nucleosome resolution is yet to be performed. Such data can provide experimental evidence to confirm or disapprove existing models or inspire new models that characterize the chromatin organization with higher accuracy. Additionally, with real experimental structural data, we could further explore the assembly, packaging, and morphology of the chromatin domains in situ to understand their functional significance. It has been reported that this higher-order chromatin structure potentially plays an important role in DNA-based processes, such as transcription, replication, and repair, and perhaps extends to complex processes, such as aging and diseases like cancer (36-39). As the physical entity of DNA, the chromatin higher-order structure can facilitate or hinder DNA accessibility by critical molecular factors involved in biochemical processes, including the regulation of global transcriptional patterns, and consequentially, the phenotypic plasticity of a cell (40-43).

In this paper, we utilized scanning transmission electron microscopy tomography with ChromEM staining (ChromSTEM) to resolve the 3D chromatin organization for two mammalian cell lines in-vitro. We observed that chromatin fibers fold into distinct, anisotropic packing domains in which the mass scaling follows a near-power-law relationship, indicating an internal fractal-like behavior of these higher-order structures. We further quantified the physical properties related to material transportation and gene accessibility of these domains, including chromatin volume concentration (CVC) and exposure ratio. Finally, we revealed such properties can be predicted by chromatin packing scaling and domain size, unveiling a potentially important link between the chromatin structure and functionality.

\section{Results}

\section{ChromSTEM imaging of chromatin organization in mammalian cells}

Following the ChromEM protocol reported previously, we labeled the DNA of human pulmonary adenocarcinoma epithelial (A549, Fig. 1, Mov. S1) and human fibroblast (BJ, Fig. S1, Mov S2) cells to characterize the chromatin packing behavior in two genetically distinct cell lines. After resin embedding, the labeled regions can be identified based on image contrast in bright field optical micrographs: the photo-oxidized cells appeared significantly darker than the nonphotobleached cells (Fig. 1A-C). Dual-tilt STEM tomography in HAADF mode was performed for part of the nucleus where a hetero/euchromatin interface was observed on a $100 \mathrm{~nm}$ resin section (Fig. 1D). We observed continuous variations of the image contrast inside the nucleus, unlike the near binary image contrast from the conventional EM staining method. Each tilt series was aligned with fiducial markers in IMOD and reconstructed by a penalized maximum likelihood (PLM-hybrid) algorithm in Tomopy $(44,45)$. The two sets of tomograms were combined in IMOD to suppress missing cone (Fig. 1E) artifacts (46). The final tomography (Fig. 1F) has a nominal voxel size of $2.9 \mathrm{~nm}$, with clearly resolved nucleosomes (Fig. 1G) and linker DNA (Fig. 1H). We also identified several distinct higher-order supranucleosomal structures, such as stacks and rings (Fig. 1I-1J). Examples of the full stack of tomography are shown in Mov. S1-S3. We also rendered the $3 \mathrm{D}$ volume of the chromatin in the volume viewer in FIJI (Fig. 1K-1L, Mov. S4-S9). The voxel intensity of the tomogram was used for color-coding (47). 


\section{ChromSTEM reveals chromatin packing domains with a fractal-like mass scaling behavior}

Due to its semi-flexible nature, the chromatin polymer can, in principle, adopt an infinite number of potential 3D conformations which are not conserved temporally or across cell populations (48). However, the statistical properties of the chromatin polymer can be characterized, and they are largely influenced by self-interaction and interaction with the surrounding nucleoplasmic environment. These properties are predicted to obey scaling laws, which describe how the number of monomers or, equivalently, the mass of the polymer, varies with the size of the physical space it occupies(49). Depending on the balance of the free energy of polymer-polymer compared to polymer-solvent interactions, under dilute, equilibrium conditions a homopolymer chain, where all monomers interact in the same way, is expected to exhibit a fractal structure characterized by a power-law relationship between the mass $(M)$ and the size $r$ at certain length scales: $M \propto r^{D}$, where $D$ is the packing scaling of the polymer. When the interaction of monomers with the solvent is preferred, $5 / 3<D<2$. When self-interaction is preferred, the polymer will collapse and adopt a scaling between 2 and 3 . In a good solvent, $D=5 / 3$ and the polymer adopts a self-avoiding random walk. When a polymer's self-interaction and interaction with the encompassing solvent are equally preferred, as in the case of an ideal chain in a theta solvent, $D=2$. A special case of $D$ $=3$ is the fractal globule structure. Importantly, in heteropolymer systems, when multiple monomer types alternate in sequential blocks along the linear polymer chain, for length scales above the size of the individual domains formed by blocks, $D=3$ can also indicate a random distribution of spatially uncorrelated domains.

Thus, a homopolymer in dilute conditions can adopt values of $D$ ranging between $5 / 3$ and 3 when in equilibrium. However, chromatin exists as a heteropolymer with the monomers, i.e., nucleosomes, possessing varying biochemical properties in the form of DNA modifications such as methylation, and those associated with post-translational histone modifications. Chromatin conformation can be further influenced by molecular mechanisms that impose additional topological constraints by actively inputting energy into the system. Such constraints include CTCF-cohesin- or transcription-dependent looping, interactions with nuclear lamins, and phase separation driven by chromatin-associated proteins such as HP1 $(50,51)$. Therefore, at any given point in time, chromatin conformation is determined by such active constraints in addition to the balance between chromatin-chromatin and chromatin-nucleoplasm interactions, resulting in a nonequilibrium system. Additionally, chromatin occupies a significant volume fraction within the nucleus. As a result of such non-dilute conditions, polymer physics does not guarantee that the entire chromatin system can be described using the same power law-scaling relationship. In other words, there may be separate regimes with different mass scaling behavior. In general, different scaling regimes for the chromatin polymer system may exist because (1) the primary chromatin chain may exhibit different intra-chain scaling, and (2) within certain scaling regimes that define fractal domains, individual domains may be characterized by different values of $D$ or the size of the individual domains may vary, which would alter the limits of this power-law scaling regime. According to SANS experiments, the scaling behavior of the chromatin polymer may be similar to the ideal chain up to a certain length scale. SANS data has previously demonstrated a complicated picture of the bi-phasic fractal organization of chromatin, with $D$ slightly higher than 2 for length-scales smaller than $300 \mathrm{~nm}$ and $D$ approaching 3 for larger scales (52). As the SANS 
experiments were performed on fixed nuclei dissolved in suspension, the scaling behavior of chromatin in vitro is still unknown.

To elucidate the chromatin structure within the cell nucleus, we investigated the mass scaling of the binary masks of chromatin segmented from the tomograms (Fig. 2A-D). In the analysis, we treat the heterogeneous chromatin fibers, as reported by Ou et al. using ChromEMT, to be the fundamental element in building higher-order structures (17). The details of the segmentation procedure can be found in Fig. S2. Practically, the 3D mass scaling relationship is defined as how the total amount of chromatin $(M)$ enclosed within a volume $\left(V=\frac{4}{3} \pi r^{3}\right)$ changes with its radius $r$. The 2D case can be described as a slice of the 3D system by a horizontal plane. In this case, $M$ is the amount of chromatin enclosed within an area $\left(A=\pi r^{2}\right)$. The derivative of the area results in the perimeter, which represents the $1 \mathrm{D}$ case. Therefore, in the $1 \mathrm{D}$ scenario, $M$ is the amount of chromatin positioned on the circumference of a circle $(P=2 \pi r)$. We refer to the 1D case as "ring mass scaling". We calculated the ring, 2D and 3D mass scaling of a fractal polymer system by performing linear regression in the log-log scale on the mass scaling curves for the given dimensions. Since the packing scaling for different dimensions can be approximated from each other for the same fractals structure by the law of additivity of fractal codimensions $(53,62)$, we confirmed from our calculations that the 3D mass scaling exponent can be estimated using the 2D and ring mass scaling (supplementary methods).

As ChromSTEM provides only a snapshot of the chromatin conformation at a single time point, we randomly sampled different regions within the field of view and calculated the mean mass scaling to capture the statistical behavior. For four A549 cells with a total volume of $1.16 \mu \mathrm{m}^{3}$ resolved at a voxel resolution of 2.0 to $2.9 \mathrm{~nm}$, we obtained the mass scaling curves at all three dimensions (Fig. 2E, F). A total volume of $0.09 \mu \mathrm{m} 3$ was reconstructed from four BJ cells at a nominal voxel resolution of 1.8 to $2 \mathrm{~nm}$ and mass scaling analysis was performed (Fig. $2 \mathrm{G}$ ). In order to obtain the packing scaling and identify length scales where a single scaling exponent cannot sufficiently describe the packing behavior, we evaluated the derivative of the log-log scale of the 3D and 2D mass scaling curves as a function of $r$. The slope, $D_{\log }$ was defined as a linear regression fit to the log-log scale of the mass scaling curves that has an error of less than $5 \%$. This linear regression fit, $D_{\text {log }}$ should be equivalent to the packing scaling, $D$ within the power-law scaling regime, which we define when length-scales associated with $D_{l o g}$ extend across at least one order of magnitude. From our 3D mass scaling analysis on A549 cells, we observed the fractal regime extending from $2 \mathrm{~nm}$ to $90 \mathrm{~nm}$ with a fitting parameter of $D_{l o g}=2.59 \pm 0.02$ (Fig. 2E, blue dashed line). However, because of the maximum section thickness of $180 \mathrm{~nm}$ for our A549 cells, our 3D analysis was unable to evaluate potential mass scaling behavior above $100 \mathrm{~nm}$. Additionally, we did not perform the 3D mass scaling analysis for BJ cells, as the thickness of the reconstructed section of $\mathrm{BJ}$ cells is smaller than $70 \mathrm{~nm}$, and the 3D mass scaling curve would only extend up to $35 \mathrm{~nm}$.

Due to the intrinsic length-scale limitation of 3D mass scaling because of limited section thickness, we then performed the mass scaling analysis at different dimensions for both A549 and BJ cells. Employing the law of additivity of fractal codimensions, we calculated the 3D mass scaling exponent from 2D and 1D mass scaling curves as (Fig. S3A): $D_{3 D}=D_{2 D}+1$, and $D_{3 D}=D_{1 D}+$ $2(53,62)$. For both A549 and BJ cells, we first evaluated the slope of the $2 \mathrm{D}$ mass scaling curve in the log-log scale along its entire length using a $12 \mathrm{~nm}$ sliding window. By estimating the local 
slope for small ranges of $r$ along the entire length of the 2D mass scaling curves, two distinct regimes were identified. The first regime stretched up to $r \sim 90 \mathrm{~nm}$, followed by a gradual increase in the local log-log derivative towards a value of 3. Similar to the 3D mass scaling analysis, for A549 cells (Fig. 2F), we then obtained the slope of linear regression, $D_{l o g}=2.60 \pm 0.01$ for $2 \mathrm{~nm}$ $<r<88 \mathrm{~nm}$ (blue dashed line). Above these length scales $(r \sim 90 \mathrm{~nm})$, the slope continuously increases until it approaches 3 for $r>252$ (red dashed line) up to $300 \mathrm{~nm}$. Similarly, for BJ cells (Fig. 2G), the fitting parameter for the linear regression was estimated to be $D_{\text {log }}=2.62 \pm 0.01$ (blue dashed line) for $2 \mathrm{~nm}<r<86 \mathrm{~nm}$, and $D_{\log }$ approaches 3 (red dashed line) for $r>182 \mathrm{~nm}$. $D_{\log }$ extending from 2 to $\sim 90 \mathrm{~nm}$ (i.e. across one order of magnitude) is indicative of a mass-fractal structure at these smaller length-scales. The shift from the fractal domain regime to the supradomain regime $\left(D_{\log } \sim 3\right)$ is continuous, as opposed to a sharp, biphasic transition. The implications of this result on the conformation of chromatin within packing domains and a detailed investigation of the boundary of the fractal regime will be discussed later.

In addition, the ring mass scaling curve exhibits a third regime from $2 \mathrm{~nm}<\mathrm{r}<10 \mathrm{~nm}$ for both cell lines (Fig. S3B-C), which can be interpreted as the chromatin chain regime. The upper length scale (10 $\mathrm{nm}$ in radius) agrees with the upper limit of the primary chromatin chain size (24 $\mathrm{nm}$ maximum diameter) (17). However, this regime is elusive on the mass scaling curves of higher dimensions, possibly caused by limited tomography resolution.

Therefore, both the 3D and 2D mass scaling analyses suggest that for length scales up to $90 \mathrm{~nm}$, chromatin packing domains with internal fractal-like structure $(D<3)$ may exist. From 2D mass scaling analyses, at larger supra-domain length scales, a gradual increase in $D$ towards a value of 3 can potentially be explained by either a variability of packing domain sizes or by an overlap between domains that are being averaged out in the mass scaling analysis. To test these hypotheses, we mapped the spatial distribution of packing scaling $D$ for the fractal regime from $2 \mathrm{D}$ mass scaling curves calculated within a moving window (300 pixels $x 300$ pixels) on each virtual 2D tomogram for each cell. We then evaluated the radial density and mass scaling profile of the identified domains as a function of distance from the center of the domains. Overlap between neighboring individual domains can be identified by a gradual increase in $D_{\log }$ from the $D$ of fractal domains to $D_{\text {log }} 3$ within the overlapping region. For both A549 (Fig. 2H) and BJ (Fig. 2I) cells, we observed domain-like structures with the mass scaling within the domains following a near power-law relationship with the packing scaling less than 3 and a sharp transition to the supradomain regime with $D_{\log } \sim 3$. This result is consistent with the scenario in which chromatin folds into spatially separable packing domains all of which exhibit internal fractal-like mass scaling behavior but differ in their genomic and physical sizes as well as the value of the mass density scaling.

\section{Quantifying domain size and chromatin packing behavior at the domain boundary}

It is worth mentioning that a mass fractal can be either deterministic or random (34). Deterministic fractal structures can be constructed from a specified element according to the deterministic rule. If the construction of a fractal structure is in any way random, the resulting fractal is random as well. For a deterministic fractal, self-similarity is manifested in that the structure is repeated on different scales. For a random mass fractal, although the structures are statistically equivalent, they can have different exact configurations at different scales. As the center of the mass scaling is 
confined within the "domain center region", we interpret the length scales as the physical distance to the "domain center region". At small length scales within this "center region", chromatin resembles a random mass fractal structure. The boundary of this fractal structure, equivalent to the size of the domain, can be defined as the length scale where the statistical behavior, such as the mass scaling, of the chromatin deviates significantly from the statistical behavior of the chromatin within the "center region". At the same time, for an isolated mass fractal with $D<3$, the chromatin density decreases from the "center region" to the periphery. For spatially separable domains which exhibit distinct mass fractal behavior, the radial chromatin density per domain is expected to initially decrease, followed by a recovery due to the intersection with other domains. Thus, the boundary of a single domain can also be dictated by the radial chromatin density profile distribution.

Our previous analysis averaged mass scaling behavior from all domains analyzed within a given field of view. Next, we wanted to better characterize the mass-scaling behavior of individual domains. To begin this more detailed analysis, we first identified the "domain center region" of each packing domain. From the spatial distribution of the packing scaling (Fig. 3A), we applied Gaussian filtering and local contrast enhancement before segmentation (Fig. 3B). Regions with the top $10 \%$ of the elevated $D$ values were included as the "domain center region" (green areas in Fig. 3B). For each domain, we resampled the mass scaling curves with centers inside the "domain center region" (Fig. 3C) and determined mass scaling behavior from these "domain centers" up to $r=400 \mathrm{~nm}$ for A549 cells and $r=200 \mathrm{~nm}$ for BJ cells. In each individual domain, the mass scaling curve exhibits regimes characterized by a gradual deviation from the initial power-law at larger length scales (Fig. 3D). We performed linear regression on the 2D mass scaling curve and obtained a slope, $D_{\text {log }}=2.71 \pm 0.02$ for $2 \mathrm{~nm}<r<102 \mathrm{~nm}$ (Fig. 3D, blue dashed line). This power-law scaling relationship can model the mass scaling curve with less than $5 \%$ error within the given fitting range, while a more significant divergence is observed beyond $r=102 \mathrm{~nm}$ (Fig. 3D, red asterisk). Therefore, from the mass scaling curve for a single packing domain, we observe that the smaller length scales have a packing scaling $D<3$, representing a mass-fractal, and that as $r$ increases up to around $100 \mathrm{~nm}$, there is a sharp transition to the supra-domain regime with $D_{\text {log }}=3$. This increase in $D$ towards a value of 3 potentially indicates overlap between neighboring domains.

Additionally, we calculated the radial distribution of chromatin volume concentration (radial CVC, supplementary methods) to investigate the chromatin packing density from the "domain center region" to the periphery of the individual domain (Fig. 3E). We observed three key trends in the radial CVC at different distances from the domain center: 1) a relatively flat, slowly decreasing curve near the domain center, 2) a rapidly decreasing curve at a moderate distance from the domain center, and 3) an increasing curve at even larger distances. This third trend is likely caused by the inclusion of chromatin from other nearby domains. The transition point from rapid decrease to increase in radial CVC (red asterisk in Figure 3E) is consistent with the transition point in mass scaling from mass fractal to random packing (red asterisk in Figure 3D), and both are indicative of the edge of the analyzed domain.

For each domain, we quantified the fractal regime boundary $\left(R_{f}\right)$ as the smallest length scale that satisfies the following criteria (Fig. S4): (1) Mass scaling curve deviates from the initial fractal power-law calculated from small length scales by $5 \%$, suggesting a significantly different packing behavior; (2) Local packing scaling $D$ reaches 3 , implying a random non-fractal structure; (3) The 
absolute value of the second derivative of the logarithm of the mass scaling curve is greater than 2, indicating a divergence from power-law scaling behavior; (4) The radial CVC starts to increase. We observed a broad range of $R_{f}$ for both A549 cells $\left(R_{f}=101.3 \pm 50.0 \mathrm{~nm}\right)$ and BJ cells $\left(R_{f}=\right.$ $89.5 \pm 36.4 \mathrm{~nm})($ Fig. $3 \mathrm{~F})$. We interpreted $R_{f}$ as the length scale where the chromatin mass scaling no longer follows a power-law relationship, or where a single packing scaling is not sufficient to explain the packing behavior. This view does not indicate each domain is spherical with radius $R_{\mathrm{f}}$. In fact, the shape of the domain boundary is not at all spherical. We further quantified the shape of the domain structure by calculating the asphericity parameter $\left(A_{s}\right)$ of the chromatin chains enclosed by the domain boundary (53). As can take on values from 0 to 1 , with 0 indicating an isotropic or spherical configuration, and 1 indicating a linear or stretched configuration. In order to avoid edge effects, we only considered domains that are entirely within the field of view. We estimated the average of $A_{s}$ to be $0.457 \pm 0.059$ from 114 domains for A549 cells and $0.458 \pm$ 0.039 from 18 domains for BJ cells, respectively (Fig. 3G). Altogether, we conclude that the packing of chromatin fibers into random mass fractal domains is heterogeneous and anisotropic.

\section{Differential morphological properties of chromatin packing domains}

With an internal mass fractal structure of $D<3$, chromatin packing domains are not space-filling with homogenous density distributions. This porous structure provides additional surface area, which could potentially promote diffusion and targeted search mechanisms, such as transcription. On the other hand, the frequency of interaction between remote genomic loci may increase with a higher fractal dimension of packing domains and higher compaction of the chromatin, due to a decrease in spatial distance between the loci. We have previously demonstrated that chromatin packing scaling, $D$, and contact probability scaling are inversely related, indicating that as packing scaling increases within a domain, the contact probability decreases at a slower rate with increasing genomic distance between two loci (56). Characterizing the distribution of such properties and understanding their link with chromatin packing can help decode the complex chromatin structurefunction relationship (43).

First, we employed the average CVC per domain to quantify the chromatin compaction. Similar to the anisotropy analysis, we excluded the domains at the edge of the field of view. We observed that $95 \%$ of 124 domains (110 domains for A549 and 14 domains for BJ) have a CVC within $24 \%$ to $49 \%$, with a mean CVC of $36.6 \%$ (Fig. 4A). For the same domains, we obtained the distribution of packing scaling $D$, with a mean value of $2.57 \pm 0.01$ for A549 cells and $D=2.65 \pm 0.03$ for BJ cells (Fig. 4B). For a mass-fractal, the CVC can be calculated from mass scaling by $C V C=\frac{M_{f}}{V_{f}}=A\left(\frac{R_{f}}{R_{\min }}\right)^{D-3} \propto A R_{e f f}^{D-3}$, where the total mass of a domain $M_{f}=A M_{\min }\left(\frac{R_{f}}{R_{\min }}\right)^{D}$ and is measured as the number of pixels occupied by chromatin within the domain, $R_{f}$ and $V_{f}$ are the domain size and total volume, $R_{\min }$ and $M_{\min }$ are the radius and the mass of the elementary unit of the chromatin chain, respectively, $A$ is the packing efficiency factor, and $R_{e f f}=\frac{R_{f}}{R_{\min }}$ is the effective domain size (56). In this description $A$ represents the chromatin packing efficiency of fundamental chromatin units within a domain. A chromatin polymer with $A=1$ specifies that each concentric layer of the packing domain is packed in the most efficient manner designated by the domain packing scaling, with the chromatin chain as the primary building block. Here, we assume that the packing efficiency within the elementary unit of the chromatin chain is 1 , i.e., the entire volume of the chain is completely filled by chromatin. Similar to $R_{f}, R_{\min }$ can be estimated from 
the ring mass scaling curve as the upper bound of the chromatin chain regime, or, in other words, the spatial separation that significantly deviates from the behavior within the chromatin chain (supplementary methods). It is of interest to investigate whether the packing efficiency and the chain size are conserved across a population of isogenic cells or across cell lines with differential genetic makeup. We investigated the relationship between domain CVC and $R_{\text {eff }}^{D-3}$ and observed a positive correlation between CVC and $D$ for A549 cells (linear regression $\mathrm{r}^{2}=0.53$ ) and a weaker correlation for BJ cells (linear regression $\mathrm{r}^{2}=0.41$ ) (Fig. 4E). Given that $R_{\min }$ from the mass scaling analysis was not significantly different across domains, cells within the same cell lines, and even between the two cell lines, this relationship suggests that the chain size may be constant across genetically different cells, although the packing efficiency is domain specific. Average $A$ for each cell line can be evaluated from the regression of CVC on $R_{\text {eff }}^{D-3}$. Comparing the A549 and BJ cell lines, we observed a significant difference between the slopes of linear regression (ANCOVA $\mathrm{p}<$ 0.0005). In conclusion, different domains have heterogeneous packing efficiencies within the same cell line, but the difference across the cell lines is even larger.

Most transcriptional processes occur on the surfaces of chromatin. Next, we studied how the probability of chromatin elements being exposed on the surface of chromatin changes across domains and across cell lines. We defined an exposure ratio $(E R)$ as the fraction of ChromSTEM voxels on the surface of the domain compared to the total number of pixels encompassing the domain volume. The surface here exclusively refers to the internal surface created by the interchromatin voids within domains. First, we define $A_{s p}$ as the surface packing efficiency, i.e. the prefactor in the scaling relationship $S_{f}=A_{s p} S_{\min }\left(\frac{R_{f}}{R_{\min }}\right)^{D-1}$ where $S_{f}$ is the total intradomain surface area and $S_{\min }$ is the surface area of the elementary unit of the chromatin chain, measured as the number of pixels. For a packing domain with a mass fractal structure, $E R$ can be then estimated by the following: $E R=\frac{\frac{s_{f}}{s_{\min }}}{\frac{M_{f}}{M_{\min }}}=A_{E R} R_{e f f}{ }^{-1}$, where $A_{E R}=\frac{A_{s p}}{A}$ is the exposure ratio efficiency factor. Next, we investigated if $A_{E R}$ is constant for all domains within the same cell line and across different cell lines. We calculated the ER for each domain and the corresponding inverse effective domain size, $R_{e f f}$. Although the distribution of $R_{e f f}$ is relatively similar between the cell lines (Fig. 4C), the variability of exposure ratios within each cell line is markedly different (Fig. 4D). Next, we performed linear regression analysis to better characterize the relationship between the inverse effective domain size and ER at the domain level. We observed a strong positive association between the $E R$ and $R_{e f f}{ }^{-1}$ for A549 cells (linear regression $\mathrm{r}^{2}=0.66$ ) and a weak, negative correlation for BJ cells (linear regression $\mathrm{r}^{2}=0.33$ ) (Fig. 4F). This suggests that for A549, $A_{E R}$ varies around a common average value but is not universal for all domains. On the other hand, the incongruent trend for the $\mathrm{BJ}$ cells suggests that $A_{E R}$ might be significantly different across cell lines.

\section{Discussion}

Utilizing ChromEM staining that selectively enhances the contrast of DNA and dual-axis electron tomography with high-angle annular dark-field imaging mode, ChromSTEM has the advantage of resolving chromatin packing in 3D at a sub-3-nm spatial resolution at the single-cell level (Fig.1). Employing ChromSTEM on two genetically different cell lines, both chemically fixed A549 cells 
(cancer) and BJ cells (non-cancer), we were able to quantify interphase chromatin packing in vitro down to the level of individual nucleosomes. Importantly, we studied these cell lines to distinguish basic principles behind chromatin packing that are generally cell line-invariant and cell linespecific. We do not assume that the exact results from A549 cells extend to all cancerous cells and the results obtained from BJ cells represent all non-cancerous cells. By analyzing the mass-scaling behavior of the chromatin polymer, we observed spatially separable and geometrically anisotropic packing domains $\sim 100 \mathrm{~nm}$ in radius in both cell lines (Fig. 2\&3). The mass scaling within the packing domains follows a power-law relationship with scaling less than 3 , which indicates that chromatin adopts a mass fractal conformation within packing domains and that packing domains have radially arranged layers with decreasing chromatin density from the domain center to the periphery. This "core-shell" structure supports earlier experimental work using super-resolution microscopy at a coarser spatial resolution.

As a random mass-fractal, the packing domains are intrinsically not space-filling. This morphology is also validated by the fact that CVC values are always smaller than $100 \%$. In addition, the local density progressively decreases from the domain center region to the periphery, which is another property of a mass-fractal domain and agrees with findings using other microscopic studies (54). At the same time, the domains are not completely isolated from each other without any chromatin density in between, as CVC values are always above 0. From these observations, it is reasonable to suggest packing domains can be considered as complex porous chromatin networks which are connected by dilute chromatin fibers.

Our previous experiments on isogenic cell lines have demonstrated $D$ as a crucial modulator of transcriptional plasticity (43). However, in these experiments either the expression levels of a certain chromatin-associated gene were altered, or a cell population was treated with a $D$-lowering agent, both while keeping other transcriptional regulators fairly similar. In general, cell lines from cancer patients which have been immortalized and propagated over generations cannot be considered genetically similar to normal, non-cancer human cells. Thus, this comparison cannot be directly extended to cell lines with distinct germline profiles such as A549 (cancer) and BJ (non-cancer). In this study, we obtained the distribution of packing scaling $D$, with mean values of $2.57 \pm 0.01$ for A549 cells and $D=2.65 \pm 0.03$ for BJ cells (Fig. 4B). In this case, other factors in addition to $D$ may be playing a role in influencing the transcriptional plasticity of these two genetically distinct cell lines, such as the alteration in the availability of transcriptional machinery, making a direct comparison extremely convoluted. $(42,56)$. Furthermore, for packing domains of both A549 and BJ cell lines, we observed a diverse range of CVC, $R_{f}, A_{s}$, and $E R$ values, all of which could also impact transcription rate (Fig. 4). As domains adopt a mass fractal packing structure, some of these morphological properties are interrelated from a polymer physics perspective. From ChromSTEM data, we observed a moderately positive correlation between $R_{e f f}{ }^{D-}$ ${ }^{3}$ and CVC for A549 cells and BJ cells, while the ratio of $R_{e f f}{ }^{D-3}$ and CVC, defined as the packing efficiency factor, varies for each individual domain. We also observed a similar relationship

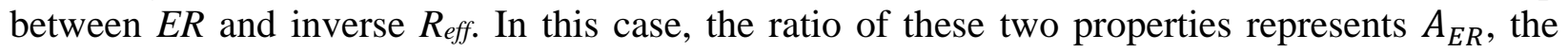
exposure ratio efficiency factor. We observed that $A_{E R}$ is not universal for all domains within a cell line and varies significantly across cell lines. The differential properties of domains could potentially play a role in regulating gene activities by controlling the size of proteins and other macromolecular complexes that can navigate through this network, thus influencing material transportation and gene accessibility. 
Outside of packing domains, the packing scaling increases to 3 after crossing the domain boundary, potentially indicating a random distribution of multiple domains with respect to each other. This arrangement is consistent with the predictions of earlier SANS experiments on different cell lines (52). On top of the agreement between SANS and ChromSTEM, there is one discrepancy in the details of how the transition occurs. In most SANS experiments, the transition of fractal dimension of $2<D<3$ for $r<300 \mathrm{~nm}$ to $D \approx 3$ at larger length scales is bi-phasic, characterized by the abrupt change in the slope of the correlation curve. In ChromSTEM, from average mass scaling analysis of all domains within a given field of view, we observed a smooth transition as evidenced by a gradual increase of the mass scaling coefficient, $D_{\log }$ at larger length scales. While the SANS data focus on the ensemble average from millions of isolated nuclei, our ChromSTEM results are purely analyzed at the single-cell level in situ. Different sample preparation methods could also be an explanation for this discrepancy.

The major limitations of ChromSTEM include chemical fixation, low throughput due to electron tomography, and inability to obtain loci-specific gene accessibility information. Therefore, ChromSTEM findings are not directly comparable to discoveries made from sequencing-based techniques such as Hi-C or loci-based imaging methods such as Fluorescence In Situ Hybridization (FISH). Despite the limitations, we believe that ChromSTEM and the associated analysis methods developed in this work should become an important tool for the understanding of the 3D structure of chromatin and its function. Additionally, we have recently demonstrated that the Nanoscale Chromatin Imaging and Analysis (nano-ChIA) platform allows quantification of different aspects of the chromatin structure by combining high-resolution imaging using ChromSTEM with other nanoimaging techniques involving labeling of molecular functionality and high-throughput chromatin dynamics imaging in live cells. Future work to extend our current understanding of chromatin structure and its relationship to gene activities should focus on developing novel labeling methods that target particular genes that are compatible with ChromSTEM sample preparation and imaging, and colocalizing chromatin morphological and genetic information for a greater number of cells from different cell lines (56).

\section{Materials and Methods}

\section{Cell culture}

A549 cells were cultured in Dulbecco's Modified Eagle Medium (ThermoFisher Scientific, Waltham, MA, \#11965092). BJ cells were cultured in Minimum Essential Media (ThermoFisher Scientific, Waltham, MA, \#11095080. All cells were maintained at physiological conditions (5\% $\mathrm{CO}_{2}$ and $37^{\circ} \mathrm{C}$ ). Experiments were performed on cells from passage 5-20.

\section{ChromEM sample preparation}

The ChromSTEM sample staining and resin-embedding followed the published protocol (58), and detailed reagents and steps can be found in Table S1. All cells were thoroughly rinsed in Hank's balanced salt solution without calcium and magnesium (EMS) before fixation with EM fixative. Two stages of fixation were performed: room temperature fixation for $5 \mathrm{~min}$ and on-ice fixation for an hour with fresh fixative. The cells were kept cold for all following steps before resin embedding either on ice or a cold stage with the temperature monitored to vary from $4^{\circ} \mathrm{C}$ to $10^{\circ} \mathrm{C}$. The biopsy of the mouse ovary was embedded in low melting point agarose (Thermo Fisher) and 
$40 \mu \mathrm{m}$ thick sections were prepared using a vibratome (VT1200 S Leica) on ice. The sections were deposited onto a glass-bottom petri-dish (MatTek) and treated as described for cells in the following steps.

After fixation, the samples were bathed in blocking buffer for $15 \mathrm{~min}$ before being stained by DRAQ5 $^{\mathrm{TM}}$ (Thermo Fisher) for $10 \mathrm{~min}$. The cells were rinsed and kept in the blocking buffer before photo-bleaching and submerged in 3-5' -diaminobenzidine (DAB) solution (Sigma Aldrich) during photo-bleaching on the cold stage.

A Nikon microscope (Nikon Inc.) was used for photo-bleaching. A cold stage was developed inhouse from a wet chamber equipped with humidity and temperature control. After photobleaching, the cells were rinsed in $0.1 \mathrm{M}$ sodium cacodylate buffer thoroughly. Reduced osmium solution (EMS) was used to enhance the contrast in STEM HAADF mode, and the heavy metal staining lasted $30 \mathrm{~min}$ on ice. Serial ethanol dehydration was performed, and during the last 100\% ethanol wash, the cells were brought back to room temperature. Durcupan resin (EMS) was used for embedding after infiltration, and the blocks were cured at $60^{\circ} \mathrm{C}$ for $48 \mathrm{hrs}$.

An ultramicrotome (UC7, Leica) was employed to prepare sections of different thicknesses. For STEM HAADF tomography, semi-thick sections were made and deposited onto a copper slot grid with carbon/Formvar film. All TEM grids were plasma cleaned before sectioning and no poststaining was performed on the sections. $10 \mathrm{~nm}$ colloidal gold fiducial markers were deposited on both sides of the sample.

A step-by-step protocol can be found in Protocol S1.

\section{EM data collection and tomography reconstruction}

A 200kV STEM (HD2300, HITACHI) with HAADF mode was employed for all image collection. For tomography, the sample was tilted from $-60^{\circ}$ to $60^{\circ}$ with $2^{\circ}$ increments on two roughly perpendicular axes. Each tilt series was aligned with fiducial markers in IMOD and reconstructed using Tomopy $(44,45)$ with a penalized maximum likelihood for 40 iterations independently. IMOD was used to combine the tomograms to suppress artifacts (Fig. S5), the nominal voxel size varies from $1.8 \mathrm{~nm}$ to $2.9 \mathrm{~nm}$ for different samples. Volume Viewer in FIJI was employed for surface rendering (59).

\section{Chromatin mask segmentation and mass scaling analysis}

We generated binary masks for chromatin from the ChromSTEM tomograms based on automatic thresholding in FIJI as reported previously with fine-tuned imaging processing parameters. For all chromatin masks used in this work, the following procedure was performed. First, the local contrast of the tomograms was enhanced by CLAHE, with a block size of 120 pixels. Then, Ostu's segmentation algorithm with automatic threshold was employed. Finally, we removed both dark and bright outliers using a threshold of 50 and a radius of 2 to refine the chromatin mask.

In polymer physics, the mass-scaling is the relationship between the material $M$ within concentric circles of radius $r$. For a fractal structure, the mass scales as $M(r) \propto r^{D}$, where $D$ is the powerscaling exponent or fractal dimension. In the 1D scenario, $M$ is the amount of chromatin positioned on the perimeter of a circle $(P=2 \pi r)$. We referred to the 1D case as "ring mass scaling". For 2D, 
$M$ is the amount of chromatin enclosed by a circle with area $A=\pi r^{2}$; for 3D, $M$ is the amount of chromatin within a sphere with volume $V=\frac{4}{3} \pi r^{3}$. To calculate mass scaling, starting from the binarized chromatin mask, concentric circles with radius from 1 pixel to 300 pixels were employed with non-zero centers were randomly selected within the sample, and $M$ is calculated as the total number of non-zero voxels within the predefined space (perimeter, area, and volume). For each stack of tomography, we averaged mass scaling curves with different centers at each dimension. For A549 cells, 1D, 2D, and 3D mass scaling were analyzed. For BJ cells, only 1D and 2D mass scaling were evaluated, as the thickness of the tomography is limited. We found from our calculations from polymer trajectories that the 3D mass scaling exponent can be approximated using the $2 \mathrm{D}$ case and the $1 \mathrm{D}$ case (Fig. S6): $D_{3 D}=D_{2 D}+1$, and $D_{3 D}=D_{1 D}+2$, with standard errors of the mean of 0.023 and 0.019 respectively.

\section{Domain and boundary analysis}

Unlike the mass scaling for the entire sample where we averaged randomly sample mass scaling curves from different regions mentioned above, to identify domains, we used the local relationship between mass scaling curves and $r$. To obtain a smooth mapping of the packing scaling, we calculated the mass scaling within a moving window (300 pixels x 300 pixels) with a stride of 1 pixel on each virtual 2D tomogram. To remove noise at small length scales, we sampled all the non-zero pixels as the center of the mass scaling analysis within the center of the window (10 pixels by 10 pixels) and obtained the average. We then calculated the packing scaling $D$ from the averaged mass scaling curve per window by linear regression, then mapped the value to the center of the window.

The above method provided the spatial distribution of packing scaling, though it is capable of identifying domains and locating the domain center region, it is not sufficient to isolate domain boundary precisely nor provide accurate values for each domain, as the moving window approach is equivalent to performing filtering. To obtain domain boundary, we calculated the mass scaling again but centered within each domain center.

The domain center is calculated from the $D$ map from the moving average method (Fig. S7). The starting point of the analysis is the $D$ map calculated by the moving window mass-scaling in grayscale. Then we applied Gaussian filtering with radius $=5$ pixels followed by CLAHE contrast enhancement with a block size of 120 pixels in FIJI(59). With a "flooding" algorithm in the MATLAB image segmentation GUI, we identified the center of the domains (green) at automatic thresholding values and created the binary mask for those regions accordingly. We then identified the center pixel of gravity per binary domain center. To obtain the mass scaling curve for a single domain, we first sampled multiple mass scaling curves with centers on the nonzero pixels around the center pixel within a 10-pixel x 10-pixel window. We then used the average mass scaling curve for that domain.

To obtain the domain boundary, we utilized the mass scaling behavior of the packing from the center region to the periphery. To evaluate such behavior, besides the mass scaling curve, we also leveraged the radial volume chromatin concentration (CVC). We adopt the definition of CVC from published work (58): which is the fraction of volume occupied by chromatin. This value is calculated from the binary chromatin mask obtained from tomography data. The boundary of the domain can be seen as the length scale where a single power-law relationship no longer holds is 
defined as the domain size $R_{f}$. Practically, we used the smallest length scale that meets at least one of the four criteria: 1. Mass scaling curve deviates from the initial fractal power-law calculated from small length scales by $5 \%$, suggesting a significantly different packing behavior; 2 . Local packing scaling $D$ reaches 3 , implying a random non-fractal structure; 3 . The absolute value of the second derivative of the logarithm of the mass scaling curve is greater than 2 , indicating a divergence from the power-law. 4. The radial CVC starts to increase. An example of the workflow to determine $R_{f}$ can be found in Fig. S4. Similar to $R_{f}$, the average size of the fundamental building block of the domain $R_{\min }$ can be measured by the spatial separation where the mass scaling behavior deviates significantly $(5 \%)$ from the behavior within the initial chromatin chain regime. In this work, the initial mass scaling behavior is quantified by the slope of the first two data points on the 2D mass scaling curve. The ratio between $R_{f}$ and $R_{\min }$ is defined as the effective domain size $R_{\text {eff. }}$

\section{Domain morphological property analysis}

We calculated four different morphological properties for each domain: packing scaling $D$, asphericity $A_{s}$, domain $\mathrm{CVC}$, and exposure ratio (ER). Chromatin packing scaling as $D_{\log }$ was estimated by the slope of the linear regression of the average mass scaling curve in log-log, fitted from $\mathrm{r} \sim 10 \mathrm{~nm}$ to $\mathrm{r} \sim 30 \mathrm{~nm}$. Chromatin within $R_{f}$ distance from the domain center pixel was selected as a "domain", though realistically the domains are likely to adopt an irregular shape. The asphericity $A_{s}$ is calculated slice-by-slice using the following expression: $A_{s}=\frac{\left(\lambda_{1}-\lambda_{2}\right)^{2}}{\lambda_{1}{ }^{2}+\lambda_{2}{ }^{2}}$, where $\lambda_{1}$ and $\lambda_{2}$ are the eigenvalues of the 2D gyration tensor of the domain (53). Then we calculated the mean value from each slice to be the $A_{s}$ for that domain. The CVC is calculated as the ratio of the total number of nonzero (chromatin) voxels over the total number of voxels per domain. And the exposure ratio is the fraction of voxels on the domain surface. The surface of the domain includes only the surface of the pores within the domain, it excludes the external surface created by an artificial boundary imposed by $R_{f}$.

\section{Protocol S1. Sample preparation for ChromSTEM for cell cultures}

\section{Fixation:}

1. Wash the cells in the petri-dish in the washing solution for 3 times, 2 minutes each.

2. Fix the cells with the fixation solution for 5 minutes at room temperature.

3. Continue to fix the cells with fresh fixation solution for an additional 1 hour on ice.

The following steps before the last ethanol dehydration are either on ice or a cold stage, all reagents must be chilled to $4^{\circ} \mathrm{C}$ before use.

\section{DNA Staining:}

4. Wash the cells with $0.1 \mathrm{M}$ sodium cacodylate buffer for 5 times on the ice, 2 minutes each.

5. Block the cells with a blocking solution for 15 minutes.

6. Stain the cells with DNA staining solution for 10 minutes.

7. Wash the cells with the blocking solution 3 times, 5 minutes each.

\section{Photo-bleaching:}

8. Bath the cells in the bathing solution before photo-bleaching 
9. Photo-bleach the cells using continuous epi-fluorescence illumination (150 W Xenon Lamp) with Cy5 red tilter and a 100x objective for 7 minutes for each spot on the cold stage.

10. Replace the bathing solution in the petri-dish with a fresh bathing solution every 15 minutes (roughly two spots).

\section{Heavy metal staining:}

11. Rinse the cells with $0.1 \mathrm{M}$ sodium cacodylate buffer 5 times, 2 minutes each.

12. Stain the cells with reduced osmium staining solution for 30 minutes.

13. Wash the cells with double distilled water 5 times, 2 minutes each.

\section{Dehydration and Resin embedding:}

14. Dehydrate the cells with serial ethanol $(30 \%, 50 \%, 70 \%, 85 \%, 95 \%, 100 \%$ twice) on ice, 2 minutes each.

15. Wash the cells with $100 \%$ ethanol at room temperature for 2 minutes.

16. Infiltrate the cells with a 1:1 infiltration mixture at room temperature for 30 minutes.

17. Infiltrate the cells with a 2:1 infiltration mixture at room temperature for 2 hours.

18. Infiltrate the cells with Durcupan ${ }^{\mathrm{TM}}$ resin mixture 1 at room temperature for 1 hour.

19. Infiltrate the cells with Durcupan ${ }^{\mathrm{TM}}$ resin mixture 2 at $50^{\circ} \mathrm{C}$ in the dry oven for 1 hour. Flat embed the cells with fresh Durcupan ${ }^{\mathrm{TM}}$ resin mixture 2 in Beem capsule and cure at $60{ }^{\circ} \mathrm{C}$ in the dry oven for 48 hours.

\section{Acknowledgment}

This work was supported by Medical Scientist Training Program T32GM008152, National Institutes of Health grants U54CA193419, R01 CA228272, R01CA225002, and National Science Foundation grants EFMA-1830961 and EFMA-1830969. This work made use of the EPIC facility of Northwestern University's NUANCE Center, which has received support from the SHyNE Resource (NSF ECCS-2025633), the International Institute for Nanotechnology (IIN), and Northwestern's MRSEC program (NSF DMR-1720139). The imaging methodology is partially based on research sponsored by the Air Force Research laboratory under agreement number is FA8650-15-2-5518. The U.S. Government is authorized to reproduce and distribute reprints for Governmental purposes notwithstanding any copyright notation thereon. The views and conclusions contained herein are those of the authors and should not be interpreted as necessarily representing the official policies or endorsements, either expressed or implied, of Air Force Research Laboratory or the U.S. Government.

\section{Author Contributions}

Y.L., V.P.D., and V.B. conceived the study, Y.L. and V.A. performed the ChromSTEM experiments. Y.L., V.A., and R.K.V analyzed the data. A.E. and J.F. assisted with the ChromSTEM experiments. E.R. and R.B. participated in the sectioning of ChromSTEM samples. K.H. and L.A. participated in the data analysis. I.S., V.P.D., and V.B. supervised the project. All authors reviewed and edited the manuscript.

\section{Conflict of Interest}


The authors declare that they have no conflict of interest.

\section{Reference}

1. J. E. Phillips-Cremins et al., Architectural protein subclasses shape 3D organization of genomes during lineage commitment. 153, 1281-1295 (2013).

2. E. J. Clowney et al., Nuclear aggregation of olfactory receptor genes governs their monogenic expression. Cell 151, 724-737 (2012).

3. W. A. Bickmore, The spatial organization of the human genome. Annual review of genomics and human genetics 14, 67-84 (2013).

4. C. A. Davey, D. F. Sargent, K. Luger, A. W. Maeder, T. J. Richmond, Solvent mediated interactions in the structure of the nucleosome core particle at $1.9 \AA$ resolution. Journal of molecular biology 319, 1097-1113 (2002).

5. D. E. Olins, A. L. J. N. r. M. c. b. Olins, Chromatin history: our view from the bridge. Nat Rev Mol Cell Bio 4, 809-814 (2003).

6. R. D. Kornberg, Y. Lorch, Twenty-five years of the nucleosome, fundamental particle of the eukaryote chromosome. Cell 98, 285-294 (1999).

7. C. L. Woodcock, R. P. J. C. S. H. p. i. b. Ghosh, Chromatin higher-order structure and dynamics. Cold Spring Harb Perspect Biol 2, a000596 (2010).

8. P. J. Horn, C. L. Peterson, Chromatin higher order folding--wrapping up transcription. Science 297, 1824-1827 (2002).

9. A. S. Belmont, S. Dietzel, A. C. Nye, Y. G. Strukov, T. J. C. o. i. c. b. Tumbar, Large-scale chromatin structure and function. 11, 307-311 (1999).

10. A. S. Belmont, K. J. T. J. o. c. b. Bruce, Visualization of G1 chromosomes: a folded, twisted, supercoiled chromonema model of interphase chromatid structure. 127, 287-302 (1994).

11. J. Finch, A. J. P. o. t. N. A. o. S. Klug, Solenoidal model for superstructure in chromatin. 73, 1897-1901 (1976).

12. A. S. Belmont, J. W. Sedat, D. A. J. T. J. o. c. b. Agard, A three-dimensional approach to mitotic chromosome structure: evidence for a complex hierarchical organization. 105, 7792 (1987).

13. M. Eltsov, K. M. MacLellan, K. Maeshima, A. S. Frangakis, J. J. P. o. t. N. A. o. S. Dubochet, Analysis of cryo-electron microscopy images does not support the existence of 30-nm chromatin fibers in mitotic chromosomes in situ. 105, 19732-19737 (2008).

14. Y. Joti et al., Chromosomes without a 30-nm chromatin fiber. 3, 404-410 (2012).

15. E. Fussner et al., Open and closed domains in the mouse genome are configured as 10-nm chromatin fibres. 13, 992-996 (2012).

16. M. A. Ricci, C. Manzo, M. F. García-Parajo, M. Lakadamyali, M. P. J. C. Cosma, Chromatin fibers are formed by heterogeneous groups of nucleosomes in vivo. 160, 11451158 (2015).

17. H. D. Ou et al., ChromEMT: Visualizing 3D chromatin structure and compaction in interphase and mitotic cells. 357, eaag0025 (2017).

18. K. Maeshima, S. Ide, M. J. C. o. i. c. b. Babokhov, Dynamic chromatin organization without the 30-nm fiber. 58, 95-104 (2019). 
19. S. V. Razin, A. A. J. E. Gavrilov, Chromatin without the 30-nm fiber: constrained disorder instead of hierarchical folding. 9, 653-657 (2014).

20. J. C. Hansen et al., The 10-nm chromatin fiber and its relationship to interphase chromosome organization. 46, 67-76 (2018).

21. K. Maeshima, S. Hihara, M. J. C. o. i. c. b. Eltsov, Chromatin structure: does the 30-nm fibre exist in vivo? 22, 291-297 (2010).

22. K. Maeshima, S. Ide, K. Hibino, M. J. C. o. i. g. Sasai, development, Liquid-like behavior of chromatin. 37, 36-45 (2016).

23. T. Nozaki et al., Dynamic organization of chromatin domains revealed by super-resolution live-cell imaging. 67, 282-293. e287 (2017).

24. M. Cremer et al., Initial high-resolution microscopic mapping of active and inactive regulatory sequences proves non-random 3D arrangements in chromatin domain clusters. 10, 39 (2017).

25. L. Tan, D. Xing, C.-H. Chang, H. Li, X. S. J. S. Xie, Three-dimensional genome structures of single diploid human cells. 361, 924-928 (2018).

26. J. R. Dixon et al., Topological domains in mammalian genomes identified by analysis of chromatin interactions. Nature 485, 376 (2012).

27. S. S. Rao et al., A 3D map of the human genome at kilobase resolution reveals principles of chromatin looping. 159, 1665-1680 (2014).

28. T. Sexton et al., Three-dimensional folding and functional organization principles of the Drosophila genome. Cell 148, 458-472 (2012).

29. Q. Szabo et al., TADs are 3D structural units of higher-order chromosome organization in Drosophila. Science Advances 4, eaar8082 (2018).

30. B. Bintu et al., Super-resolution chromatin tracing reveals domains and cooperative interactions in single cells. Science 362, eaau1783 (2018).

31. L. A. J. C. r. Mirny, The fractal globule as a model of chromatin architecture in the cell. 19, 37-51 (2011).

32. A. L. Sanborn et al., Chromatin extrusion explains key features of loop and domain formation in wild-type and engineered genomes. 112, E6456-E6465 (2015).

33. S. Wang et al., Spatial organization of chromatin domains and compartments in single chromosomes. 353, 598-602 (2016).

34. E. Iashina, S. J. J. o. e. Grigoriev, t. physics, Large-Scale Structure of Chromatin: A Fractal Globule or a Logarithmic Fractal? 129, 455-458 (2019).

35. K. Huang et al., Physical and data structure of 3D genome. 6, eaay4055 (2020).

36. G. Li, D. J. C. o. i. g. Reinberg, development, Chromatin higher-order structures and gene regulation. 21, 175-186 (2011).

37. J. G. Wood, S. L. J. F. i. g. Helfand, Chromatin structure and transposable elements in organismal aging. 4, 274 (2013).

38. T. J. C. S. H. p. i. b. Misteli, Higher-order genome organization in human disease. 2 , a000794 (2010).

39. D. G. Lupiáñez, M. Spielmann, S. J. T. i. G. Mundlos, Breaking TADs: how alterations of chromatin domains result in disease. 32, 225-237 (2016).

40. L. M. Almassalha et al., Macrogenomic engineering via modulation of the scaling of chromatin packing density. Nature biomedical engineering 1, 902 (2017).

41. L. M. Almassalha et al., The global relationship between chromatin physical topology, fractal structure, and gene expression. 7, 41061 (2017). 
42. H. Matsuda, G. G. Putzel, V. Backman, I. Szleifer, Macromolecular crowding as a regulator of gene transcription. Biophysical journal 106, 1801-1810 (2014).

43. R. Virk et al., Disordered Chromatin Packing Regulates Ensemble Gene Expression and Phenotypic Plasticity. 118, 549a-550a (2020).

44. J. R. Kremer, D. N. Mastronarde, J. R. McIntosh, Computer visualization of threedimensional image data using IMOD. Journal of structural biology 116, 71-76 (1996).

45. D. Gürsoy, F. De Carlo, X. Xiao, C. Jacobsen, TomoPy: a framework for the analysis of synchrotron tomographic data. Journal of synchrotron radiation 21, 1188-1193 (2014).

46. D. N. Mastronarde, Dual-axis tomography: an approach with alignment methods that preserve resolution. Journal of structural biology 120, 343-352 (1997).

47. J. Schindelin et al., Fiji: an open-source platform for biological-image analysis. 9, 676-682 (2012).

48. C. Zimmer, How the genome folds: biophysics of 4D chromatin organization.

49. P.-G. De Gennes, P.-G. Gennes, Scaling concepts in polymer physics. (Cornell university press, 1979).

50. Q. MacPherson, B. Beltran, A. J. J. P. o. t. N. A. o. S. Spakowitz, Bottom-up modeling of chromatin segregation due to epigenetic modifications. 115, 12739-12744 (2018).

51. J. Nuebler, G. Fudenberg, M. Imakaev, N. Abdennur, L. A. J. P. o. t. N. A. o. S. Mirny, Chromatin organization by an interplay of loop extrusion and compartmental segregation. 115, E6697-E6706 (2018).

52. D. Lebedev et al., Fractal nature of chromatin organization in interphase chicken erythrocyte nuclei: DNA structure exhibits biphasic fractal properties. FEBS letters 579, 1465-1468 (2005).

53. F. Kenneth. (John Wiley and sons, 1990).

54. I. J. T. J. o. c. p. Szleifer, A new mean-field theory for dilute polymer solutions: Phase diagram, conformational behavior and interfacial properties. 92, 6940-6952 (1990).

55. T. Cremer et al., The interchromatin compartment participates in the structural and functional organization of the cell nucleus. 42, 1900132 (2020).

56. Li, Yue, et al. "Nanoscale chromatin imaging and analysis platform bridges 4D chromatin organization with molecular function." Science Advances 7.1 (2021): eabe4310.

57. H. D. Ou et al., ChromEMT: Visualizing 3D chromatin structure and compaction in interphase and mitotic cells. Science 357, eaag0025 (2017).

58. J. R. Kremer, D. N. Mastronarde, J. R. McIntosh, Computer visualization of threedimensional image data using IMOD. Journal of structural biology 116, 71-76 (1996).

59. D. Gürsoy, F. De Carlo, X. Xiao, C. Jacobsen, TomoPy: a framework for the analysis of synchrotron tomographic data. Journal of synchrotron radiation 21, 1188-1193 (2014).

60. J. Schindelin et al., Fiji: an open-source platform for biological-image analysis. Nature methods 9, 676 (2012).

61. I. J. T. J. o. c. p. Szleifer, A new mean-field theory for dilute polymer solutions: Phase diagram, conformational behavior and interfacial properties. 92, 6940-6952 (1990).

62. Thill, A., et al. "Determination of structure of aggregates by confocal scanning laser microscopy." Journal of colloid and interface science 204.2 (1998): 357-362. 
Table S1. Reagents used in ChromSTEM Staining

\begin{tabular}{|c|c|}
\hline Reagent & Formula \\
\hline Washing solution & $\begin{array}{l}\text { Hank's balanced salt solution without calcium and } \\
\text { magnesium }\end{array}$ \\
\hline Fixation solution & $\begin{array}{l}2.5 \% \text { EM grade glutaraldehyde } \\
2 \% \text { paraformaldehyde } \\
2 \mathrm{mM} \mathrm{CaCl}_{2} \\
0.1 \mathrm{M} \text { sodium cacodylate buffer, } \mathrm{pH}=7.4\end{array}$ \\
\hline Blocking solution & $\begin{array}{l}10 \mathrm{mM} \text { glycine } \\
10 \mathrm{mM} \text { potassium cyanide } \\
0.1 \mathrm{M} \text { sodium cacodylate buffer, } \mathrm{pH}=7.4\end{array}$ \\
\hline DNA staining solution & $\begin{array}{l}10 \mu \mathrm{M} \text { DRAQ5 } \\
0.1 \% \text { SAPONIN } \\
0.1 \mathrm{M} \text { sodium cacodylate buffer, } \mathrm{pH}=7.4\end{array}$ \\
\hline Bathing solution & $\begin{array}{l}2.5 \mathrm{mM} 3,3^{\prime} \text { - diaminobenzidine tetrahydrochloride (DAB) } \\
0.1 \mathrm{M} \text { sodium cacodylate buffer, } \mathrm{pH}=7.4\end{array}$ \\
\hline $\begin{array}{l}\text { Reduced osmium staining } \\
\text { solution }\end{array}$ & $\begin{array}{l}2 \% \text { osmium tetroxide } \\
1.5 \% \text { potassium ferrocyanide } \\
2 \mathrm{mM} \mathrm{CaCl}_{2} \\
0.15 \mathrm{M} \text { sodium cacodylate buffer, } \mathrm{pH}=7.4\end{array}$ \\
\hline Durcupan ${ }^{\mathrm{TM}}$ resin mixture 1 & $\begin{array}{l}10 \text { mL Durcupan }{ }^{\mathrm{TM}} \mathrm{ACM} \text { single component } \mathrm{A}, \mathrm{M} \text {, epoxy resin } \\
10 \mathrm{~mL} \text { Durcupan }{ }^{\mathrm{TM}} \mathrm{ACM} \text { single component } \mathrm{B} \text {, hardener } 964 \\
0.15 \mathrm{~mL} \text { Durcupan }{ }^{\mathrm{TM}} \mathrm{ACM} \text { single component } \mathrm{D}\end{array}$ \\
\hline Durcupan ${ }^{\mathrm{TM}}$ resin mixture 2 & $\begin{array}{l}10 \text { mL Durcupan }{ }^{\mathrm{TM}} \mathrm{ACM} \text { single component } \mathrm{A}, \mathrm{M} \text {, epoxy resin } \\
10 \mathrm{~mL} \text { Durcupan }{ }^{\mathrm{TM}} \mathrm{ACM} \text { single component } \mathrm{B} \text {, hardener } 964 \\
0.2 \mathrm{~mL} \text { Durcupan }{ }^{\mathrm{TM}} \mathrm{ACM} \text {, single component } \mathrm{C} \text {, accelerator } \\
960 \\
0.15 \text { mL Durcupan }{ }^{\mathrm{TM}} \text { ACM single component } \mathrm{D}\end{array}$ \\
\hline 1:1 infiltration mixture & $\begin{array}{l}10 \mathrm{~mL} 100 \% \text { ethanol } \\
10 \mathrm{~mL} \text { Durcupan }{ }^{\mathrm{TM}} \text { resin mixture } 1\end{array}$ \\
\hline $2: 1$ infiltration mixture & $\begin{array}{l}5 \mathrm{~mL} 100 \% \text { ethanol } \\
10 \mathrm{~mL} \text { Durcupan }{ }^{\mathrm{TM}} \text { resin mixture } 1\end{array}$ \\
\hline
\end{tabular}




\section{Figures Legends}

Fig. 1 ChromSTEM tomography reconstruction of the chromatin of an A549 cell. (A-B) The DRAQ5 photo-oxidation process takes $7 \mathrm{~min}$ for each region of interest. Scale bar: $10 \mu \mathrm{m}$. (C) The labeled regions were more intensely stained than the nearby regions (red squares; the letter corresponds to the regions in the left panels). Scale bar: $20 \mu \mathrm{m}$. (D) STEM image of a $100 \mathrm{~nm}$ thick section of an A549 cell in HAADF mode. Scale bar: $2 \mu \mathrm{m}$. (E) Schematics for dual-tilt tomography. The sample was tilted from $-60^{\circ}$ to $60^{\circ}$ with $2^{\circ}$ increments on two perpendicular axes. (F) 3D tomography of the A549 chromatin. Scale bar: $120 \mathrm{~nm}$. (G-H) The fine structure of the chromatin chain: Nucleosomes (blue arrows in G), linker DNA (blue arrows in $\mathbf{H}$ ) supranucleosomal stack (red dashed lines in $\mathbf{I}$ ), and ring (red dashed circles in $\mathbf{J}$ ). Scale bar: 30 $\mathrm{nm}$. (K-L) 3D rendering of the chromatin organization, the pseudo-color was based on the intensity of the tomograms (Mov. S4-S6). (L) A magnified view of the region labeled by a white square in $\mathbf{K}$. In $\mathbf{L}$, pink and green regions represent high and low DNA density regions, respectively.

Fig.2 Chromatin folds into packing domains with a fractal-like mass scaling behavior. (A) STEM HAADF image of a $150 \mathrm{~nm}$ section of a BJ cell nucleus for tomography reconstruction. Scale bar: $2 \mu \mathrm{m}$. (B) A magnified view of the chromatin in the nuclear periphery of the same cell in A with gold fiducial markers. The intensity variation of the image shows that the chromatin packs at different densities throughout. Scale bar: $200 \mathrm{~nm}$. (C) A virtual 2D slice of the chromatin of a BJ cell after tomography reconstruction. Scale bar: $100 \mathrm{~nm}$. (D) Binary mask of the chromatin location for the same area in $\mathbf{C}$. The mass scaling analysis was performed on the binary masks. (E-G) The average mass scaling (MS) curve at different dimensions of the chromatin was imaged from four A549 (E, F) and four BJ $(\mathbf{G})$ cells. The mass scaling analysis was conducted with randomly selected centers within the field of view, and a mean mass scaling curve is shown for each dimension, the error bar represents the standard deviation. (E) 3D mass scaling curve exhibits power-law behavior with a single scaling up to $r=90 \mathrm{~nm}$. Slope, $D=2.59$ \pm 0.02 was obtained from linear regression from $r=2 \mathrm{~nm}$ to $r=90 \mathrm{~nm}$. We found that the 3D mass scaling exponent can be approximated using the $2 \mathrm{D}$ case and the $1 \mathrm{D}$ case: $D_{3 D}=D_{2 D}+1$, and $D_{3 D}=D_{1 D}+2$. Two regimes of mass scaling with different packing scaling $D$ can be identified. In the $2 \mathrm{D}$ cases for both A549 cells $(\mathbf{F})$ and BJ cells $(\mathbf{G})$, the MS curve starts with a packing scaling similar to that of a mass-fractal with $D<3$ (blue dashed line) and smoothly transitions to values close to $D=3$ (red dashed line). (H) Spatial distribution of packing scaling $D$ within the fractal regime of an A549 cell. The color represents the value of $D$. Scale bar: 200 $\mathrm{nm}$. (I) The spatial distribution of $D$ within the fractal regime of a BJ cell. Scale bar: $100 \mathrm{~nm}$.

Fig.3 Chromatin packing domains are structurally heterogeneous and anisotropic. (A) The spatial mapping of packing scaling $D$ within the fractal regime for one field of view of an A549 cell. Scale bar: $200 \mathrm{~nm}$. (B) From the grayscale representation of $\mathbf{A}$ after smoothing and local contrast enhancement, the domain centers are identified by the top $10 \%$ of the $\mathrm{D}$ value. For one domain, the mass scaling curve is resampled from centers within the domain center (green region). (C) 3D rendering of the surface of chromatin fibers in a region, including the packing domain of interest (orange square in B). (D) The average 2D mass scaling (MS) curve of the chromatin within the region of interest (orange square in $\mathbf{B}$ and $\mathbf{C}$ ). The mass scaling analysis is conducted with 
randomly selected centers within the domain center. The error bar represents the standard deviation. The MS curve starts with a packing scaling similar to that of a mass-fractal with $D<3$ (blue dashed line) and transitions to values closer to $D=3$ (beyond the red asterisk). (E) Radial distribution of CVC for the same domain shown in $\mathbf{B}, \mathbf{C}$, and $\mathbf{D}$. The radial CVC initially slowly decreases within the fractal regime. As the length scale approaches the domain boundary (red asterisk), the radial CVC rapidly dips which is followed by a recovery, due to the presence of other domains at those length scales. (F) The distribution of $R_{f}$, the radius of the packing domain, for A549 (blue) and BJ (orange) cells. (G) The distribution of $\mathrm{A}_{\mathrm{s}}$, the asphericity of the chromatin fibers within the domain, for A549 (blue) and BJ (orange) cells.

Fig. 4 Differential morphological properties of chromatin packing domains. (A) Chromatin volume concentration (CVC) distribution per packing domain for A549 cells and BJ cells. A total of 110 A549 cell packing domains and 14 BJ cell packing domains were analyzed. We observed the CVC distribution ranges from 0.10 to 0.80 with a mean value of 0.37 for A549 cells, and the CVC distribution ranges from 0.19 to 0.55 with a mean value of 0.35 for BJ cells. (B) For the same domains, we observed packaging scaling $D$ varies from 2.19 to 2.80 with a mean equal to 2.57 for A549 cells and $D$ from 2.45 to 2.82 with a mean equal to 2.65 for BJ cells. (C) Effective domain size $R_{\text {eff }}$ for A549 and BJ cells. The effective domain size is the ratio between domain size $R_{f}$ and domain chain size $R_{\min }$. For A549, $\mathrm{R}_{\mathrm{f}}$ ranges from 3.4 to 23.3 with a mean value of 10.2 . For BJ, $\mathrm{R}_{\mathrm{f}}$ ranges from 3.5 to 15.0 with a mean of 7.6. (D) Exposure Ratio (ER) per domain fraction is defined as the fraction of chromatin voxels on the surface of the interchromatin voids. For A549, the ER ranges from 0.15 to 0.59 with a mean value of 0.26 . For $B J$, the ER ranges from 0.20 to 0.69 with a mean of 0.33 . (E) A moderate correlation between domain CVC and $D$ has been observed for A549 cells, with $\mathrm{r}^{2}=0.53$, and a weaker correlation with $\mathrm{r}^{2}=0.41$ for BJ cells. (F) Exposure ratio is positively correlated with inverse effective domain size with the strong linear coefficient for A549, characterized by $\mathrm{r}^{2}=0.66$, but showed a very weak negative correlation for $\mathrm{BJ}$, with $\mathrm{r}^{2}=0.33$. 
Fig.1 ChromSTEM tomography reconstruction of chromatin in an A549 cell

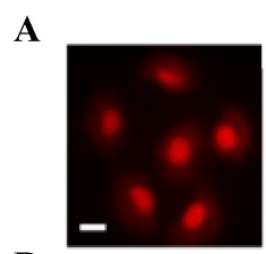

B

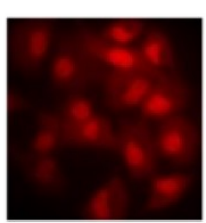

D

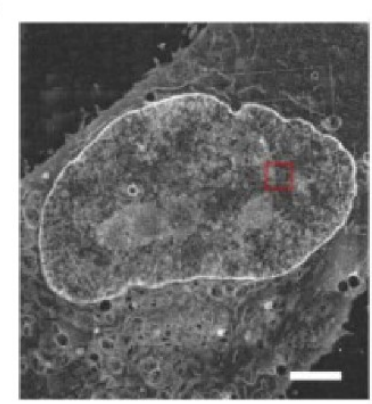

C

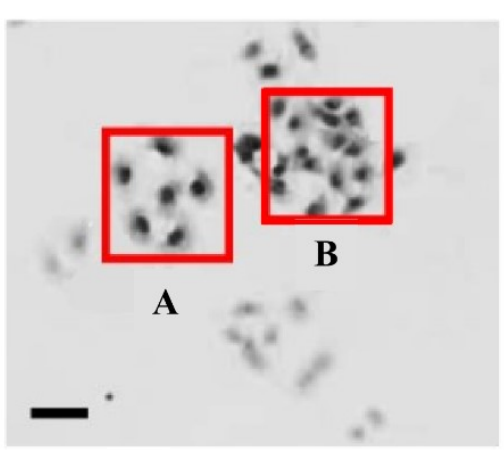

E

\section{F}

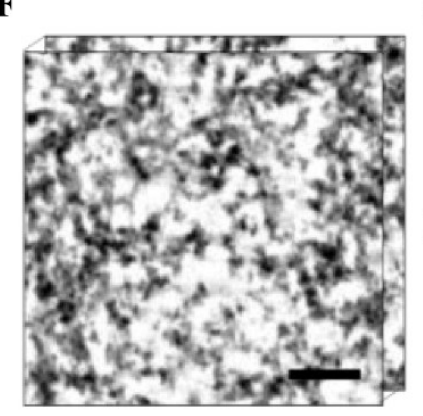

K

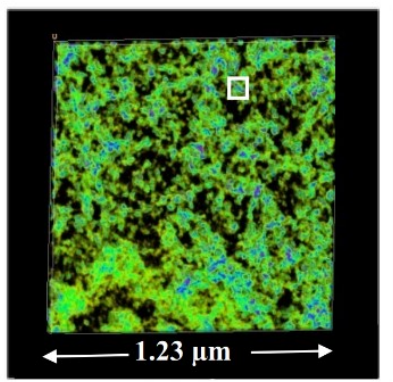

G I

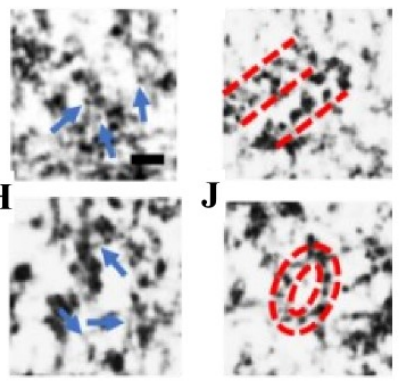

L

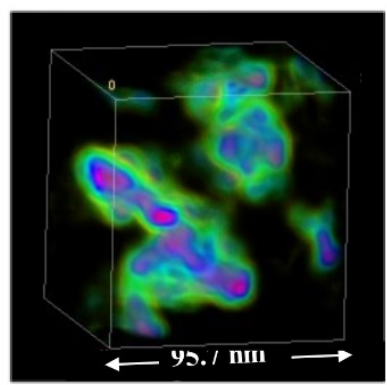


Fig.2 Chromatin folds into packing domains with a fractal-like mass scaling behavior
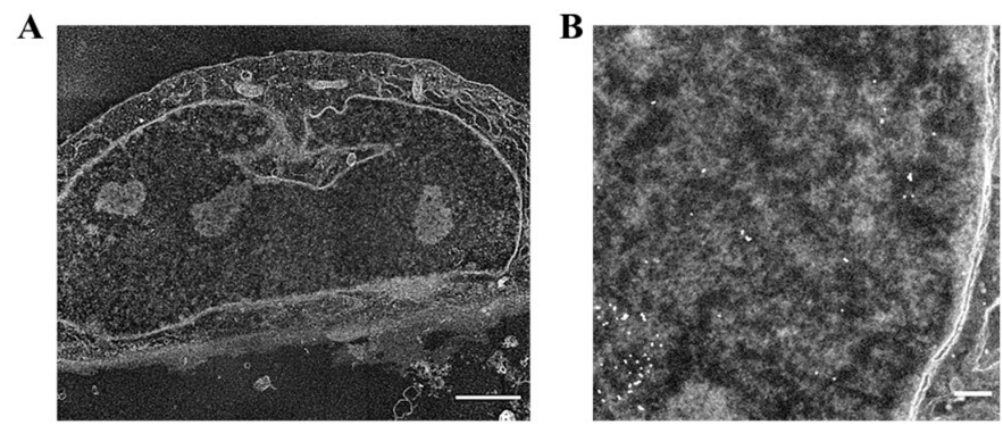

$\mathbf{C}$
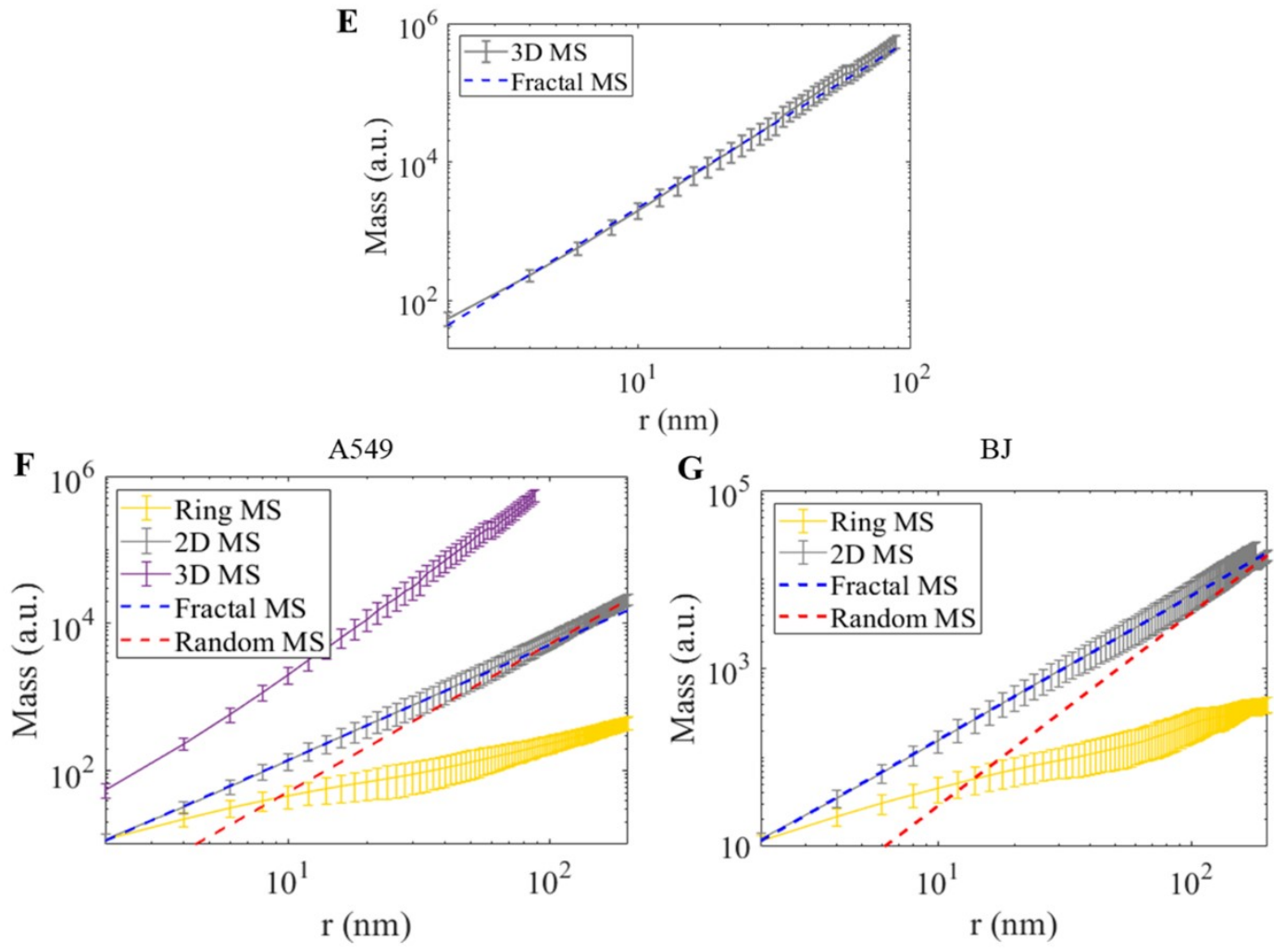
H
A549

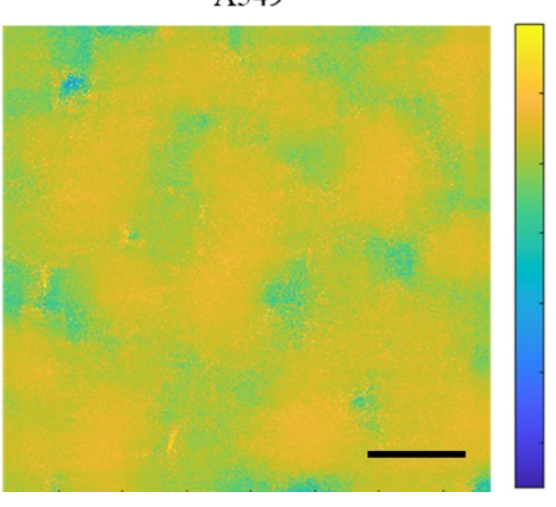

3

I

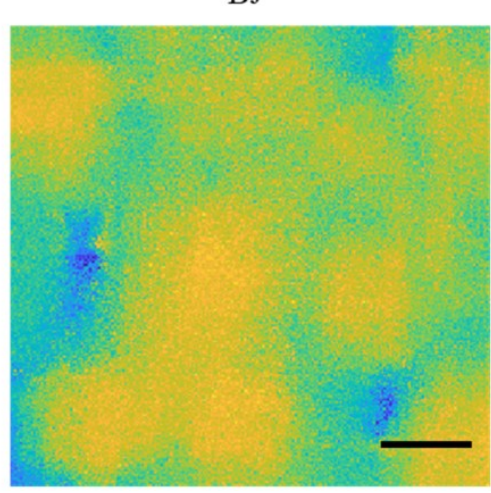

3

1.8 
Fig.3 Quantifying domain size and chromatin packing behavior at the domain boundaries

A

A A549

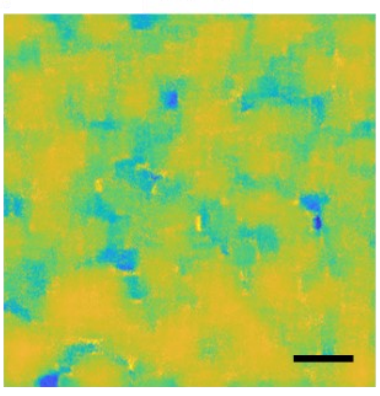

B

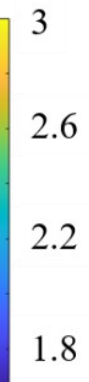

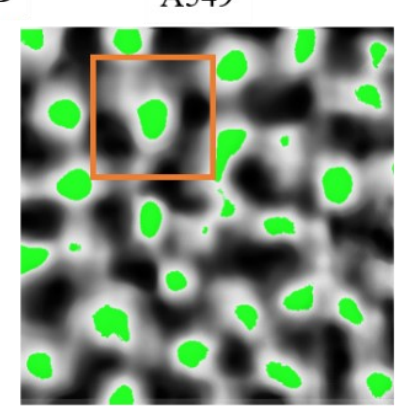

C

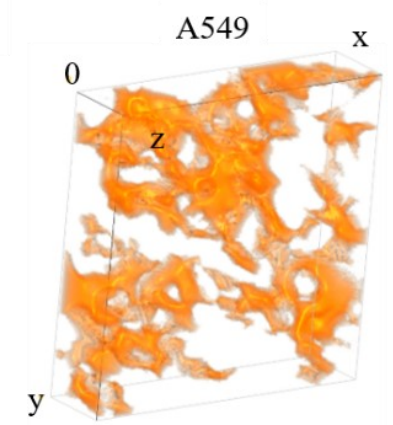

D
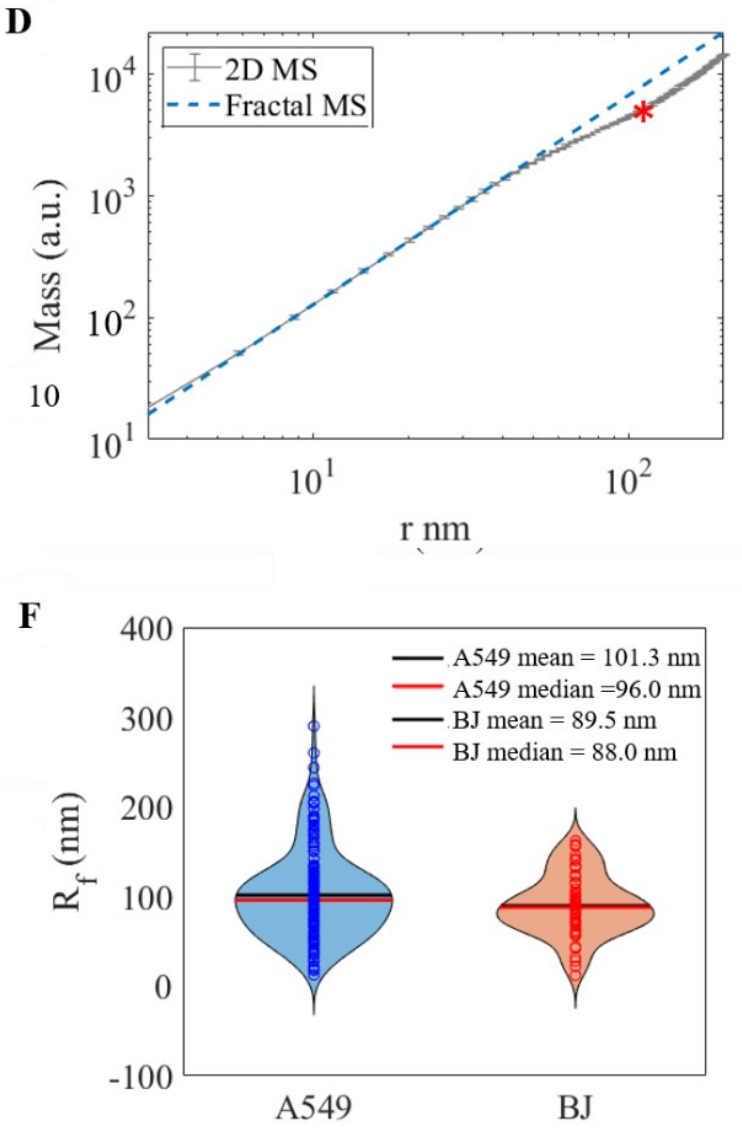

E
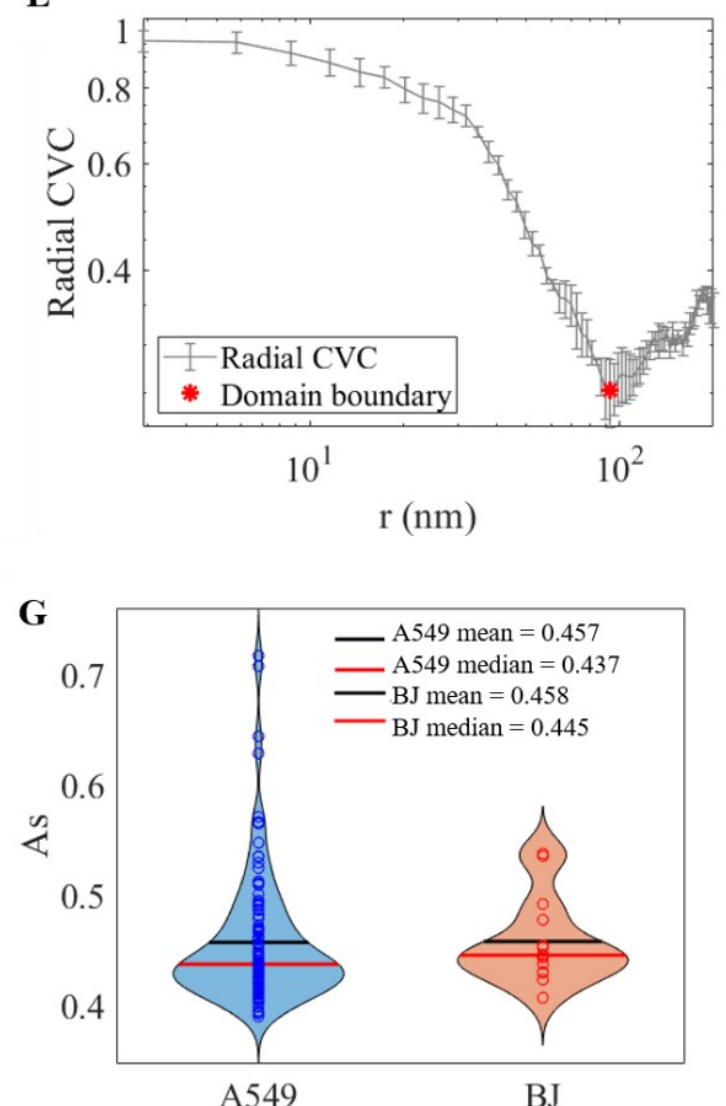
Fig. 4 Differential properties of chromatin packing domains

$\mathbf{A}$

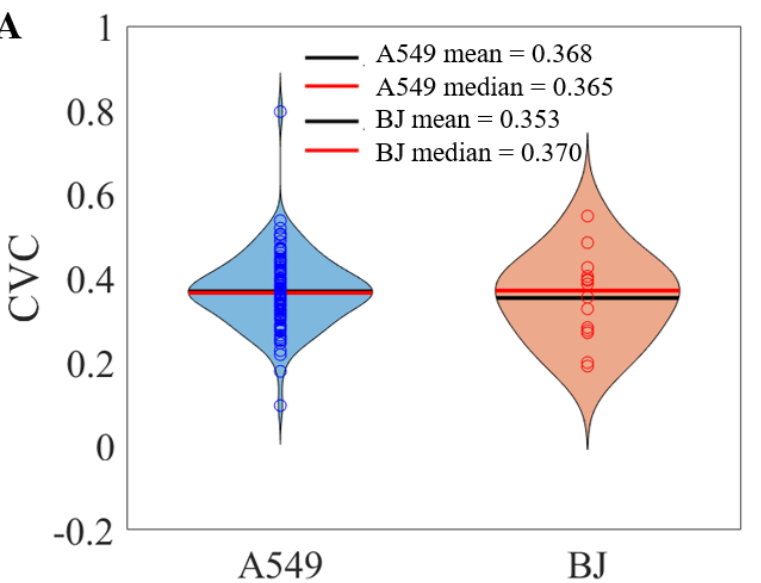

B 3.2

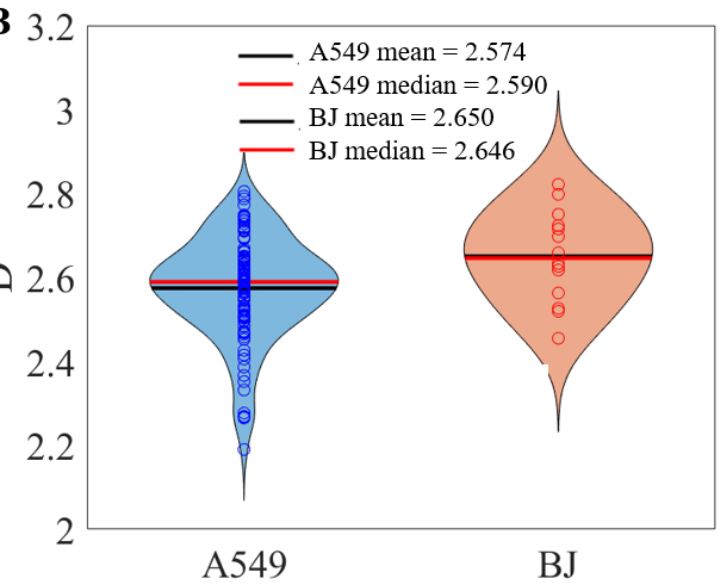

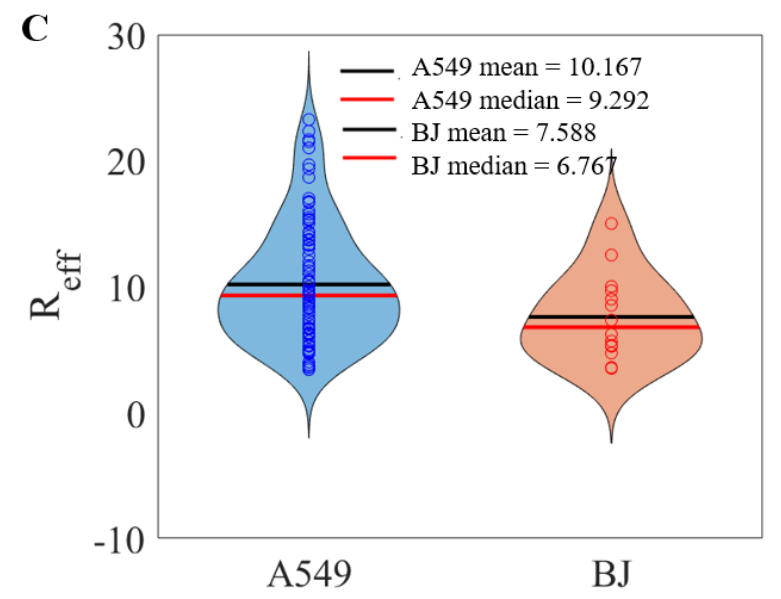
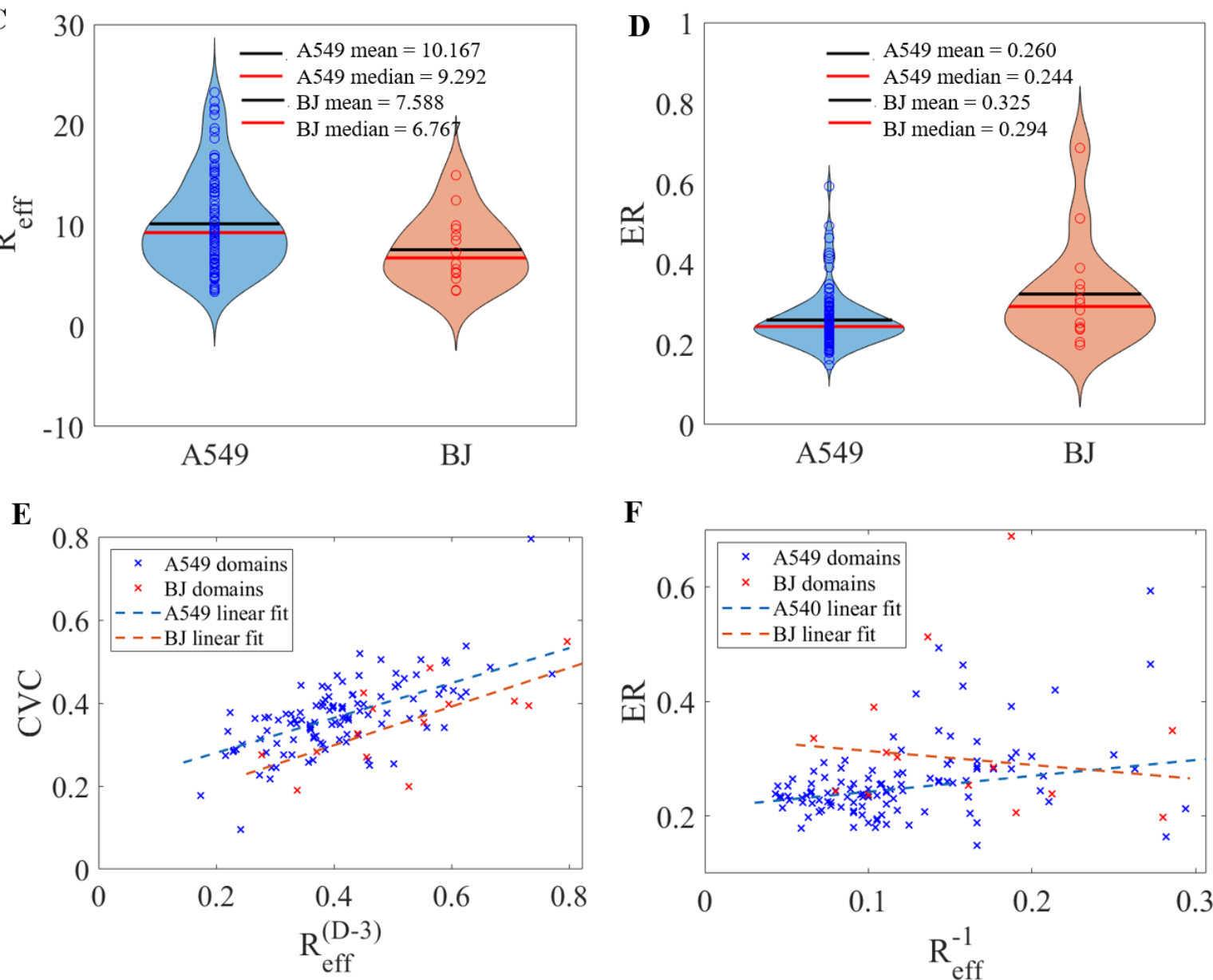

$\mathbf{F}$

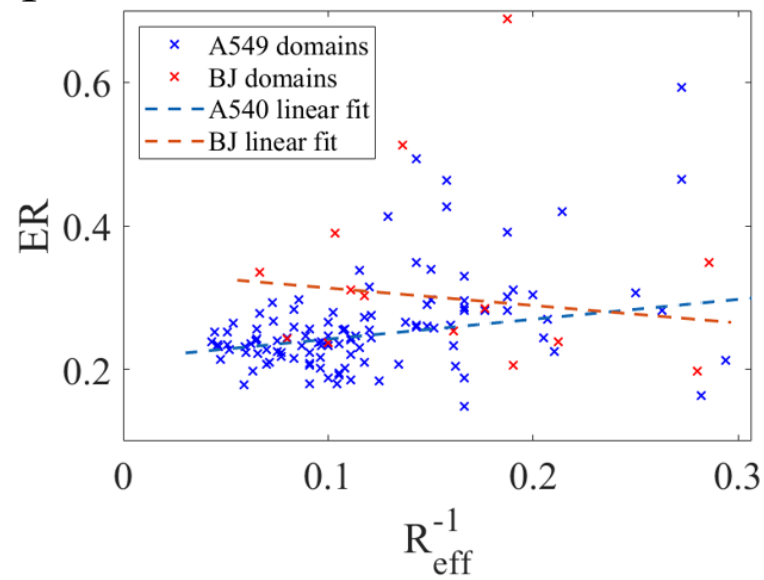

